Document downloaded from:

http://hdl.handle.net/10251/64713

This paper must be cited as:

Fouad, YMM.; Gohary, RH.; Cabrejas Peñuelas, J.; Yanikomeroglu, H.; Calabuig Soler, D.; Roger Varea, S.; Monserrat Del Río, JF. (2015). Time-frequency Grassmannian signalling for MIMO multi-channel-frequency-flat systems. IEEE Communications Letters. 19(3):475478. doi:10.1109/LCOMM.2014.2386873.

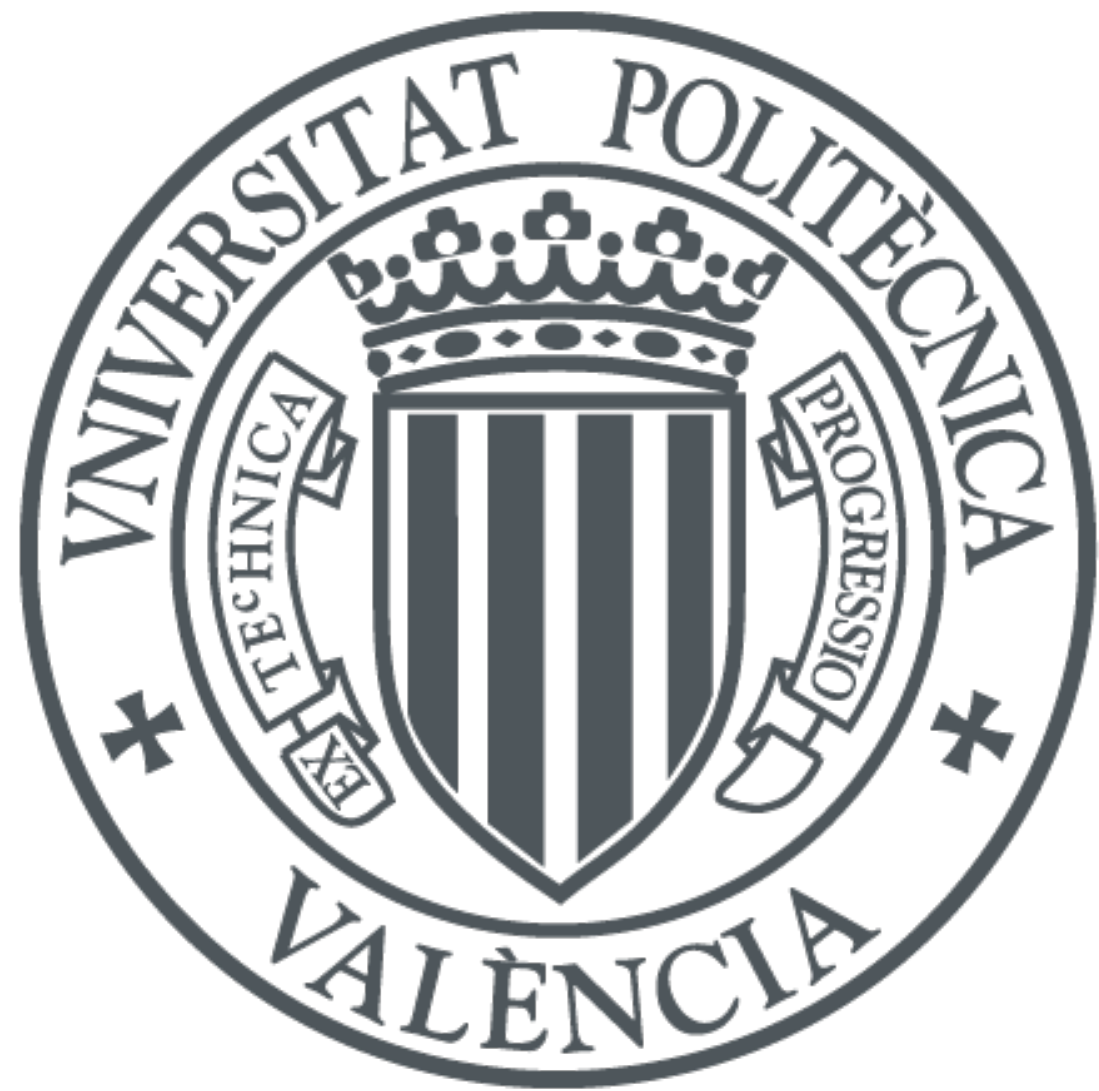

The final publication is available at

http://dx.doi.org/10.1109/LCOMM.2014.2386873

Copyright Institute of Electrical and Electronics Engineers (IEEE)

Additional Information 


\title{
Time-Frequency Grassmannian Signalling For MIMO Multi-Channel-Frequency-Flat Systems
}

\author{
Yaser M. M. Fouad, Ramy H. Gohary, Jorge Cabrejas, Halim Yanikomeroglu, Daniel Calabuig, Sandra Roger, \\ and Jose F. Monserrat
}

\begin{abstract}
In this paper, we consider the application of noncoherent Grassmannian signalling in practical multi-channelfrequency-flat multiple-input-multiple-output (MIMO) wireless communication systems. In these systems, Grassmannian signalling, originally developed for single-channel block-fading systems, is not readily applicable. In particular, in such systems, the channel coefficients are constant across time and frequency, which implies that spectrally-efficient signalling ought to be jointly structured over these domains. To approach this goal, we develop a concatenation technique that yields a spectrallyefficient time-frequency Grassmannian signalling scheme, which enables the channel coherence bandwidth to be regarded as an additional coherence time. This scheme is shown to achieve the high signal-to-noise ratio non-coherent capacity of MIMO channels when the fading coefficients are constant over a timefrequency block. This scheme is also applicable in fast fading systems with coherence bandwidth exceeding that of one subchannel. The proposed scheme is independent of the symbol duration, i.e., the channel use duration, and is thus compatible with the transmit filter designs in current systems.
\end{abstract}

\section{INTRODUCTION}

Coherent communication schemes are widely used in wireless systems including those based on the Long Term Evolution (LTE) standards. This is due in part to the simplicity of their detection and their ability to utilize standard signalling constellations [1]. Despite these advantages, coherent communication requires the channel coefficients to be known at the receiver. This is usually accomplished by sending training symbols that enable the receiver to estimate those coefficients. Unfortunately, the number of training symbols increases linearly with the number of transmit antennas [2] and is inversely proportional to the channel coherence time and bandwidth [3]. This fact results in the proliferation of training symbols and represents one of the main challenges of incorporating massive multiple-input-multiple-output (MIMO) approaches [4] in beyond 4G systems.

In addition to encroaching on available resources, trainingbased schemes have questionable efficacy. To see that, we note that wireless channels that arise in practice are usually time-varying with coefficients that are not known a priori to the receiver, that is, practical communication channels are inherently non-coherent. With this view in mind, it can be seen that the rate yielded by training-based schemes is only a lower bound on the non-coherent channel capacity [5]. Hence,

Yaser Fouad, Ramy Gohary, and Halim Yanikomeroglu are with the Department of Systems and Computer Engineering, Carleton University, Ottawa, ON, Canada. Jorge Cabrejas, Daniel Calabuig, Sandra Roger, and Jose Monserrat are with the Institute of Telecommunications and Multimedia Applications, Universitat Politècnica de València, Valencia, 46022 Spain. to avoid excessive proliferation of training symbols, and to enable higher data rates to be reliably communicated over practical communication channels, one may utilize signalling schemes that do not require explicit acquisition of instantaneous channel state information (CSI). One candidate is the Grassmannian scheme of [5], which was shown to achieve the ergodic high SNR non-coherent MIMO capacity for standard single-channel block-fading communication scenarios, that is, scenarios in which the channel coefficients on each subchannel are essentially constant over each block of channel uses, but are otherwise independent across blocks and subchannels.

In contrast with the single-channel case, in multi-channelfrequency-flat systems, the channel coefficients are constant not only in time, but also over several contiguous subchannels. For such systems, it can be shown that independent Grassmannian signalling over individual subchannels within the coherence bandwidth incurs a significant loss in the achievable rate. Hence, to enable the application of the Grassmannian construction of [5] in multi-channel-frequency-flat systems, the transmitted signals must be jointly structured over time, frequency and space, analogous to space-time-frequency coding in coherent systems [6]. To do so, we propose a technique that provides an equivalent single-channel block-fading system by concatenating the subchannels within the coherence bandwidth. This technique induces a time-frequency structure that yields a spectrally-efficient Grassmannian signalling scheme with the following favorable features.

- Achieving the high SNR non-coherent capacity of MIMO block-fading channels when the channel coefficients are constant over a time-frequency block.

- Applicability in fast fading systems with coherence bandwidth exceeding that of one subchannel.

- Compatibility with the pulse characteristics used in current systems.

\section{SySTEM MODEL}

Consider an orthogonal frequency division multiplexing (OFDM) system with $M$ transmit and $N$ receive antennas. The normalized channel coherence time and bandwidth, denoted by $T_{c}$ and $B_{c}$, represent the duration in number of channel uses and the bandwidth in number of subchannels over which the channel coefficients are essentially constant, respectively. In this paper, we refer to frequency-flat blockfading systems in which $B_{c}>1$ and $B_{c}=1$ as multichannel and single-channel, respectively. The channel use duration, $T_{s}$, spans one OFDM symbol and the subchannel bandwidth, $B_{s}$, corresponds to the bandwidth of one OFDM 
subchannel. The system utilizes Grassmannian signalling for high SNR communications over a block Rayleigh fading channel in which the coefficients assume independent realizations between consecutive blocks. For each block of duration $T_{c}$, a symbol, $\mathbf{X}^{(k)} \in \mathbb{C}^{T_{c} \times M}$, is transmitted over a subchannel $k \in\left\{1, \ldots, B_{c}\right\}$. The $(t, m)$-th entry of $\mathbf{X}^{(k)}$ represents the transmitted pulse at time $t \in\left\{1, \ldots, T_{c}\right\}$, by transmit antenna $m \in\{1, \ldots, M\}$, over subchannel $k$. In such subchannel, the channel gains matrix and the received symbol are denoted by $\mathbf{H}^{(k)} \in \mathbb{C}^{M \times N}$ and $\mathbf{Y}^{(k)} \in \mathbb{C}^{T_{c} \times N}$, respectively, where

$$
\mathbf{Y}^{(k)}=\mathbf{X}^{(k)} \mathbf{H}^{(k)}+\mathbf{W}^{(k)} \text {. }
$$

The $(t, n)$-th entry of $\mathbf{Y}^{(k)}$ represents the received pulse at time $t$, by the $n \in\{1, \ldots, N\}$ receive antenna, and $\left[\mathbf{W}^{(k)}\right]_{T_{c} \times N} \sim \mathcal{C N}(0,1)$. Although Grassmannian signalling is capacity-achieving in systems with $B_{c}=1$, it incurs a significant loss in the achievable rate when independently utilized over individual subchannels with $B_{c}>1$. This drawback can be alleviated by utilizing the techniques proposed hereinafter.

\section{NON-COHERENT GRASSMANNIAN SIGNALLING IN MULTI-CHANNEL-FREQUENCY-FLAT SYSTEMS}

Practical measurements show that, with standard subchannel bandwidths, e.g., $15 \mathrm{KHz}$ in case of LTE, the channel coefficients are constant over multiple adjacent subchannels, thereby yielding $B_{c}>1$. To see that independent Grassmannian signalling over individual subchannels incurs a loss in achievable rates, we note that the philosophy of this scheme is to impose a unitary structure across the pulses transmitted in a block within which the channel coefficients are constant. The size of this channel block increases monotonically with the number of communication degrees of freedom, $\psi$. For instance, the number of degrees of freedom of an $M$ transmit and $N$ receive antenna system utilizing Grassmannian signalling to communicate over a block-fading channel with $B_{c}=1$ and coherence time $T_{c}$ is given by $\psi_{T_{c}}=M\left(1-\frac{M}{T_{c}}\right)$. This value is the coefficient of $\log _{2}$ (SNR) in the high SNR non-coherent channel capacity expression, and is maximized by the largest integer $M$ satisfying $M \leq \min \left\{N,\left\lfloor\frac{T_{c}}{2}\right\rfloor\right\}$ [5]. In contrast, when the channel coefficients are constant over a $T_{c} \times B_{c}$ timefrequency block, the number of degrees of freedom can be seen to be $\psi_{T_{c} B_{c}}=M\left(1-\frac{M}{T_{c} B_{c}}\right)$, where $M \leq \min \left\{N,\left\lfloor\frac{T_{c} B_{c}}{2}\right\rfloor\right\}$. Hence, single-channel Grassmannian signalling results in the loss of $\frac{M^{2}}{T_{c}}\left(1-\frac{1}{B_{c}}\right)$ degrees of freedom; a drawback that can be mitigated by the two techniques proposed next.

\section{A. Reshaping of the transmitted pulse}

In multi-channel-frequency-flat systems, the underlying transmit filter can be modified to yield an equivalent singlechannel system with a longer $T_{c}$, thus enabling the efficient implementation of Grassmannian signalling. To elaborate, from a spectral perspective, since the bandwidth occupied by a pulse of duration $T_{s}, B_{s} \approx \frac{1}{T_{s}}$, modifying the system transmit filter can generate pulses with reduced durations, thereby occupying the entire coherence bandwidth and rendering $B_{c}=1$. Now, from a temporal perspective, reducing the duration of transmit pulses increases the number of channel uses spanned by $T_{c}$.

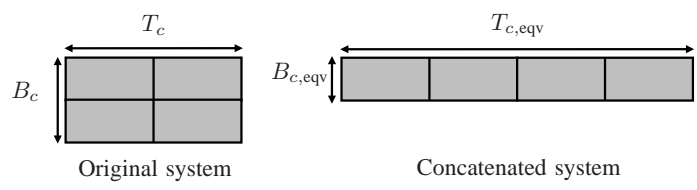

Fig. 1. Concatenation of subchannels for a system with $B_{c}=2$ and $T_{c}=2$.

Despite its advantages, reshaping the transmitted pulse has two major drawbacks in practice. First, in current OFDM cellular systems, $B_{s}$ is selected such that it ensures backward compatibility with their legacy systems; e.g., compatibility between LTE systems and their High Speed Packet Access (HSPA) predecessors is ensured by setting $B_{s}=$ $15 \mathrm{KHz}$ [7]. Second, increasing $B_{s}$ results in decreasing the OFDM symbol duration, which subsequently reduces its efficiency; i.e., the ratio of the duration of the cyclic prefix to that of the OFDM symbol [7]. Given these relatively stringent constraints on $B_{s}$, a more practical approach to efficiently utilize Grassmannian signalling in systems with $B_{c}>1$ is to implement a technique that is independent of $B_{s}$.

\section{B. Concatenated Grassmannian signalling}

In this section, we propose a technique whereby the subchannels within $B_{c}$ are concatenated to obtain an equivalent single-channel system. This concatenation does not affect the underlying subchannel bandwidths and thus it is readily applicable in current and future systems. After concatenation, the normalized channel block duration of the equivalent singlechannel system, $T_{c, \text { eqv }}=T_{c} B_{c}$. Similarly, the normalized coherence bandwidth of the equivalent system, $B_{c, \text { eqv }}=1$. An illustration of concatenation is depicted in Figure 1.

The system model of the equivalent single-channel system can be obtained by modifying the expression in (1) as follows:

$$
\overline{\mathbf{Y}}=\overline{\mathbf{X}} \mathbf{H}+\overline{\mathbf{W}},
$$

where $\overline{\mathbf{Y}}=\left[\mathbf{Y}^{(1)^{T}} \ldots \mathbf{Y}^{\left(B_{c}\right)^{T}}\right]^{T}$ is the $T_{c, \text { eqv }} \times N$ received signal matrix, $\overline{\mathbf{X}}=\left[\mathbf{X}^{(1)^{T}} \cdots \mathbf{X}^{\left(B_{c}\right)^{T}}\right]^{T}$ is the $T_{c \text {,eqv }} \times M$ transmitted Grassmannian symbol, and $\overline{\mathbf{W}}=$ $\left[\mathbf{W}^{(1)^{T}} \ldots \mathbf{W}^{\left(B_{c}\right)^{T}}\right]^{T}$ is the $T_{c \text {, eqv }} \times N$ additive white Gaussian noise matrix. Since the block-fading channel is assumed to be constant over a $T_{c} \times B_{c}$ time-frequency block, the channel coefficients matrix, $\mathbf{H}$, is constant across all subchannels within $B_{c}$; i.e., $\mathbf{H}^{(k)}=\mathbf{H}, \forall k \in\left\{1, \ldots, B_{c}\right\}$.

Having transformed the multi-channel-frequency-flat system into an equivalent single-channel one, capacity-achieving communication at high SNRs can be established using Grassmannian signalling, but in $G\left(T_{c \text {,eqv }}, M\right)$ instead of $G\left(T_{c}, M\right)$; i.e., the dimensions of each Grassmannian symbol are $T_{c \text {,eqv }} \times M$, instead of $T_{c} \times M$. However, unlike the single-channel case in which each Grassmannian symbol is sent independently over one subchannel, in the equivalent system, each Grassmannian symbol is transmitted over all the subchannels within $B_{c}$. In particular, each $T_{c, \text { eqv }} \times M$ Grassmannian symbol is partitioned into $T_{c} \times M$ submatrices each of which is then transmitted on one of the subchannels within $B_{c}$. This introduces a structure between the signal matrices transmitted over different 
subchannels, thus enabling the utilization of all the available $\psi_{T_{c} B_{c}}$ degrees of freedom. At the receiver side, the $T_{c \text {, eqv }} \times M$ signal matrix can be recovered by detecting a concatenation of the submatrices received on the $B_{c}$ subchannels.

To elaborate on the capacity-achieving property of the proposed technique, we note that it was shown in [5] that Grassmannian signalling achieves the high SNR non-coherent block-fading channel capacity of single-channel systems; i.e., it achieves the capacity of the equivalent system after concatenation. However, the concatenation underlying the proposed technique is a reversible operation that does not affect capacity. In other words, this technique enables a multi-channelfrequency-flat system to communicate at the high SNR channel capacity. This technique also enables the application of the Grassmannian in fast fading systems as elucidated next.

\section{Grassmannian signalling in Tone-Hopping OFDM systems}

In Tone-Hopping OFDM systems (TH-OFDM), users hop pseudorandomly across subchannels in each time slot thereby rendering $T_{c}=1$ [8]. Since space-time Grassmannian signalling, without exploiting the frequency dimension, requires that $M \leq\left\lfloor\frac{T_{c}}{2}\right\rfloor[5]$, this scheme is inapplicable in TH-OFDM systems. However, if the channel coefficients are constant across multiple adjacent subchannels the concatenation technique of Section III-B can be used for space-frequency Grassmannian signalling, analogous to space-frequency signalling in coherent communication scenarios [6]. The equivalent singlechannel system in that case has $T_{c, \text { eqv }}=B_{c}$ and its high SNR ergodic non-coherent capacity is maximized with $M=\left\lfloor\frac{B_{c}}{2}\right\rfloor$.

\section{NUMERICAL RESULTS}

In this section, we provide a numerical comparison between the spectral efficiency of concatenated and independent singlechannel Grassmannian signalling schemes when utilized in systems with $B_{c}>1$. We also evaluate the approximate spectral efficiency of concatenated Grassmannian signalling in an LTE Urban Micro-cell (UMi) setup with a channel delay spread, $\sigma=129 \mathrm{~ns}$ [9]. We consider a user terminal (UT) moving at a speed of $25 \mathrm{Km} / \mathrm{hr}$ in an LTE UMi and communicating at a carrier frequency of $1.8 \mathrm{GHz}$. For this UT, the Doppler frequency $f_{d}=41.67 \mathrm{~Hz}$ and the $T_{c \text {,eqv }}$ can be evaluated using the time-frequency correlation expression [10]

$$
R(\Delta t, \Delta f)=\frac{J_{0}^{2}\left(2 \pi f_{d} \Delta t\right)}{1+2 \pi(\Delta f)^{2} \sigma^{2}},
$$

where $J_{0}(\cdot)$ is the zero-order Bessel function of the first kind, and $\Delta t$ and $\Delta f$ are the signalling duration and bandwidth over which the channel correlation is evaluated. Given the LTE subchannel bandwidth, the spectral correlation $R(0, \Delta f)=$ $0.999 \approx 1$ for a block of six subchannels; i.e., a 90 $\mathrm{KHz}$ bandwidth. Similarly, the OFDM symbol duration in LTE is $71.4 \mu \mathrm{s}$, which gives rise to a temporal correlation of $R(\Delta t, 0)=0.99 \approx 1$ for a block of $T_{c}=8$ channel uses; i.e., $T_{c, \text { eqv }}=T_{c} B_{c}=48$.

We consider three channel scenarios, all with $M=N=4$ antennas and $T_{c}=8$ channel uses, but with $B_{c}=3,6$, and 10 subchannels. In Figure 2, we show the maximum achievable

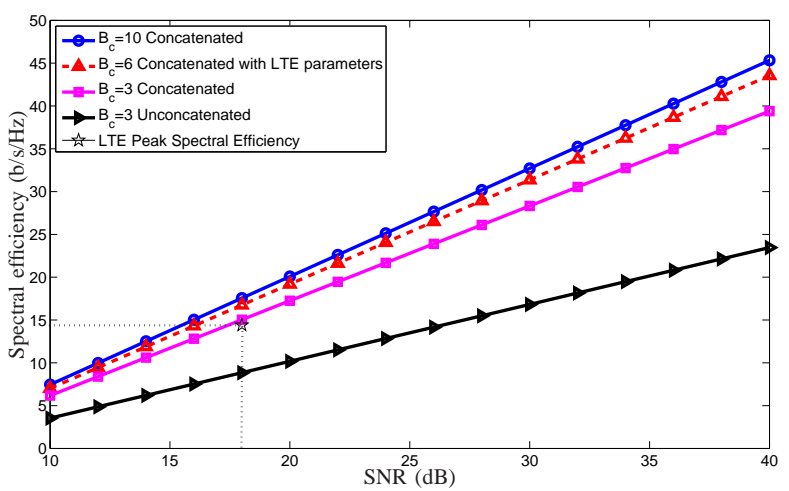

Fig. 2. Spectral efficiency of concatenated and single-channel Grassmannian with $M=N=\frac{T_{c}}{2}=4$; LTE peak spectral efficiency also depicted.

spectral efficiency by both, the single-channel and the concatenated Grassmannian schemes in a multi-channel-frequency-flat system. The dashed curve represents the approximate spectral efficiency of the concatenated Grassmannian scheme in the LTE UMi scenario. We also depict the LTE peak spectral efficiency at 64 QAM and 5/6 code rate with only the channel estimation overhead taken into consideration [11].

From Figure 2, it can be seen that the concatenated timefrequency Grassmannian signalling is always superior to the independent utilization of subchannels within $B_{c}$ in the singlechannel Grassmannian scheme of [5]. For instance, at an SNR of $30 \mathrm{~dB}$, a gain of $75 \%$ in the spectral efficiency can be observed for the case in which $B_{c}=3$ and $T_{c}=8$. This gain is due to the increase in $\psi$ and approaches $\frac{M^{2}}{T_{c}}$ for asymptotically large $B_{c}$. Furthermore, concatenated Grassmannian signalling is also superior to the LTE peak spectral efficiency. This is because, in LTE, $14 \%$ of the resources are allocated for training symbols to acquire the CSI when $M=4$. We note that the performance gains of the concatenated Grassmannian signalling can also be obtained by the pulse shaping technique, but at the expense of changing the underlying transmit filter of the OFDM system and subsequently its symbol efficiency.

\section{CONCLUSION}

We proposed a concatenation technique that yields a spectrally-efficient time-frequency Grassmannian signalling scheme for MIMO block-fading multi-channel-frequency-flat systems. The proposed technique introduces a structure between the signals transmitted over the subchannels within $B_{c}$, thus utilizing the fact that the channel coefficients are constant in both time and frequency domains. This eliminates the loss in the degrees of freedom incurred by using independent Grassmannian signalling over individual subchannels within $B_{c}$. Hence, this technique enables the system to communicate at the high SNR non-coherent MIMO block-fading channel capacity when the channel coefficients are constant over a timefrequency channel block. The proposed technique also renders Grassmannian signalling applicable in fast fading systems in which $B_{c}>1$. The proposed concatenation technique does not require changing the underlying pulse duration of the system, and is therefore compatible with current and future systems. 


\section{REFERENCES}

[1] A. Goldsmith, Wireless Communications. Cambridge: Cambridge University Press, 2005.

[2] B. Hassibi and B. M. Hochwald, "How much training is needed in multiple-antenna wireless links?," IEEE Trans. Inf. Theory, vol. 49, pp. 951-963, Apr. 2003.

[3] S. Sesia, I. Toufik, and M. Baker, LTE - The UMTS Long Term Evolution: From Theory to Practice. Great Britain: John Wiley, 2011.

[4] G. Caire, N. Jindal, M. Kobayashi, and N. Ravindran, "Multiuser MIMO achievable rates with downlink training and channel state feedback," IEEE Trans. Inf. Theory, vol. 56, pp. 2845-2866, June 2010.

[5] L. Zheng and D. N. C. Tse, "Communication on the Grassmann manifold: a geometric approach to the noncoherent multiple-antenna channel," IEEE Trans. Inf. Theory, vol. 48, pp. 359-383, Feb. 2002.

[6] W. Su, Z. Safar, M. Olfat, and K. J. R. Liu, "Obtaining full-diversity space-frequency codes from space-time codes via mapping," IEEE Trans. Signal Processing, vol. 51, pp. 2905-2916, Nov. 2003.

[7] E. Dahlman, S. Parkvall, and J. Skld, 4G LTE/LTE-Advanced for Mobile Broadband. Oxford: Elsevier Ltd., 2011.

[8] R. Laroia, S. Uppala, and J. Li, "Designing a mobile broadband wireless access network," IEEE, Signal Processing Magazine, vol. 21, pp. 20-28, Sept. 2004.

[9] ITU-R, "Guidelines for evaluation of radio interface technologies for IMT-Advanced," tech. rep., International Telecommunication Union (ITU), 2008.

[10] W. C. Lee, Mobile Communications Engineering. McGraw-Hill, 1982.

[11] T. Beitelmal, R. Schoenen, and H. Yanikomeroglu, "On the impact of correlated shadowing on the performance of user-in-the-loop for mobility," in Proc. IEEE Int. Conf. Commun. (ICC), (Ottawa), June 2012. 